\title{
On Distributed Function Computation in Structure-Free Random Networks
}

\author{
Sudeep Kamath and D. Manjunath* \\ Dept of Electrical Engg \\ IIT Bombay Mumbai INDIA 400076 \\ sudeep, dmanju@ee.iitb.ac.in
}

\begin{abstract}
THIS PAPER IS ELIGIBLE FOR THE STUDENT BEST PAPER AWARD. We consider in-network computation of MAX in a structure-free random multihop wireless network. Nodes do not know their relative or absolute locations and use the Aloha MAC protocol. For one-shot computation, we describe a protocol in which the MAX value becomes available at the origin in $O(\sqrt{n / \log n})$ slots with high probability. This is within a constant factor of that required by the best coordinated protocol. A minimal structure (knowledge of hop-distance from the sink) is imposed on the network and with this structure, we describe a protocol for pipelined computation of MAX that achieves a rate of $\Omega\left(1 /\left(\log ^{2} n\right)\right)$.
\end{abstract}

\section{Introduction}

Early work on computation of functions of binary data over wireless networks focused on computing over noisy, time-slotted, broadcast networks, e.g., [1, 6]. With increasing interest in wireless sensor networks, recent research has concentrated on 'in-network' computation over multihop wireless networks, e.g., [2, 5, 4], which also assume that time is slotted. The primary focus of the above research has been to define an oblivious protocol that identifies the nodes that are to transmit in each slot. This implies that the nodes have organized themselves into a network and have their clocks synchronized. Both of these require significant effort. In this paper we describe a protocol for in-network computation of MAX in a structure-free network, (i.e., in a network where nodes do

${ }^{*}$ Research carried out at the Bharti Centre for Communication Research and supported in part by grants from the Ministry of Information Technology of Government of India

ACCEPTED AT ISIT 2008 
not have an identity and hence do not know the topology) that uses the Aloha MAC protocol. We first describe the One-Shot MAX protocol for one-shot computation of the MAX and its analysis. We show that, with high probability (w.h.p.), the sink will have the result in a time that is within a constant factor of that required by a structured network. We then impose a minimal structure and describe the Pipelined MAX protocol and its analysis. We show that the rate of computing the MAX in this network is $\Omega\left(\frac{1}{\log ^{2} n}\right)$.

\section{MAX in Multihop Aloha}

$n$ nodes are uniformly distributed in $[0,1]^{2}$ and each node is assumed to know $n$. The sink, the node that is to have the value of the MAX, is at the origin. The nodes do not have an identity and they do not know either their relative or their absolute positions. Hence, the network does not know its topology. This of course means that a schedule for transmissions cannot be defined. Thus a random access protocol is an obvious choice at the MAC layer. We first assume that the nodes use the s-Aloha MAC protocol. For pedagogical convenience, we will assume slotted-Aloha at the MAC layer. The analysis easily extends to the case of pure Aloha MAC.

Spatial reuse is analyzed using the well-known protocol model of interference [3]. For s-Aloha, this model translates to the following. Consider a transmitter at location $x_{1}$ transmitting in a slot $t$. A receiver at location $x_{2}$, can successfully decode this transmission if and only if the following two conditions are satisfied. (1) $\left\|x_{2}-x_{1}\right\|<r_{n}$, and (2) $\left\|x_{2}-x_{3}\right\|>\left(1+\Delta^{\prime}\right) r_{n}$ for some constant $\Delta^{\prime} \geq 0 ; x_{3}$ is the location of any other node transmitting in slot $t . r_{n}$ is called the transmission radius. A transmission in slot $t$ is deemed successful if all nodes within $r_{n}$ of the transmitter receive it without collision. The following is a sufficient condition for successful transmission by a node located at $x$ in a slot: $\left\|x-x^{\prime}\right\|>(1+\Delta) r_{n}, \Delta=1+\Delta^{\prime}$, for every other node transmitting in that slot and located at $x^{\prime}$.

\subsection{One-shot computation of MAX using Aloha}

Let $Z_{i}$ be the value of the one-bit data at Node $i$ and $\mathcal{Z}:=\max _{1 \leq i \leq n} Z_{i}$. The protocol One-Shot MAX is as follows. Node $i$ can either receive or transmit in a slot but not both. In slot $t$, Node $i$ will either transmit, with probability $p$ or listen, with probability $(1-p)$, independently of all the other transmissions in the network. Let $X_{i}(t)$ be the value of the bit received (i.e., correctly decoded in the absence of a collision) by Node $i$ in slot $t, t=1,2, \ldots$. If Node $i$ transmits in slot $t$ or if it senses a collision or idle in the slot, then it sets $X_{i}(t)=0$. Define $Y_{i}(0)=Z_{i}$ and 
$Y_{i}(t):=\max \left\{Y_{i}(t-1), X_{i}(t)\right\}$ for $t=1,2, \ldots Y_{i}(t)$ is the 'running MAX' at Node $i$ in slot $t$. If Node $i$ transmits in slot $t$, it will transmit $T_{i}(t)=Y_{i}(t-1)$.

It is easy to see that the correct value of $\mathcal{Z}$ will 'diffuse' in the network in every slot. The performance of the protocol, that is, the diffusion time, depends on $p$. The choice of $p$ is discussed in Section 3 . To study the progress of the diffusion, we will consider a tessellation of the unit square into square cells of side $s_{n}=\left\lceil\sqrt{\frac{n}{2.75 \log n}}\right\rceil^{-1}$. This will result in $l_{n}:=\frac{1}{s_{n}}=\left\lceil\sqrt{\frac{n}{2.75 \log n}}\right\rceil$ rows (and columns) of cells in $[0,1]^{2}$. There will be a total of $M_{n}:=\frac{1}{s_{n}^{2}}=\left\lceil\sqrt{\frac{n}{2.75 \log n}}\right\rceil^{2}$ cells. Let $\mathcal{C}$ denote the set of cells under this tessellation. Let $S_{c}$ be the set of nodes in Cell $c$ and $N_{c}$ be the number of nodes in Cell $c$. Under this tessellation, two cells are said to be adjacent if they have a common edge. Let the transmission radius be $r_{n}=\sqrt{\frac{13.75 \log n}{n}} \approx \sqrt{5} s_{n}$. For this value of $r_{n}$ the network is connected w.h.p. [3]. The expected number of nodes in a cell is $n s_{n}^{2} \approx 2.75 \log n$. Further, from Lemma 3.1 of [7, for our choice of $r_{n}$ and $s_{n}$,

$$
\operatorname{Pr}\left(c_{1} \log n \leq N_{c} \leq c_{2} \log n \text { for } 1 \leq c \leq M_{n}\right) \rightarrow 1
$$

where $c_{1}=0.091$ and $c_{2}=5.41$. Our results will hold for networks that are connected and which satisfy (1). From the choice of $r_{n}$ (i.e., $r_{n} \geq \sqrt{5} s_{n}$ ) a successful transmission by any node from Cell $c$ is correctly decoded by all nodes in Cell $c$ as well as by all nodes in cells adjacent to Cell $c$. The value of $\mathcal{Z}$ can reach the sink along any of the many possible trees rooted at the sink. For our analysis, we will divide the progress of the diffusion into the following three 'phases' and analyze each of the three phases separately. We reiterate that the above sequence of phases is for the purpose of analysis of the time to diffusion. The nodes do not perform any such organization.

- Phase I for data aggregation within each cell. This phase is completed when every node of the network has transmitted successfully at least once.

- Phase II for progress to the bottom of the square. In this phase, the locally computed values of the MAX get diffused into the cells on one side of the unit square as shown in Fig. 1 .

- Phase III for progress into the sink. In this phase, the value of MAX reaches the sink at the origin in the manner shown in Fig. 1.

We show in Section 3 that Phase I will be completed in $O\left(\log ^{2} n\right)$ slots w.h.p., Phase II and Phase III will each be completed in $O\left(\sqrt{\frac{n}{\log n}}\right)$ slots w.h.p. These results are combined into the following theorem. 


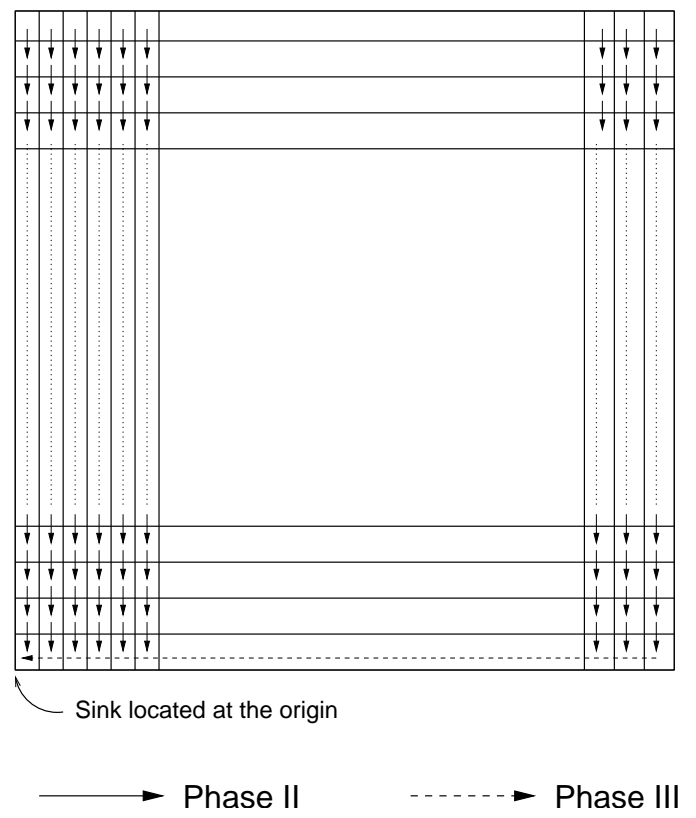

Figure 1: Direction of diffusion during Phase II and Phase III of protocol One-Shot MAX

Theorem 1 If all the nodes execute the protocol One-Shot MAX, then for any $\alpha, k>0$, the maximum of the binary data at the $n$ nodes is available at the sink with probability at least $\left(1-\frac{k}{n^{\alpha}}\right)$ in $O\left(\sqrt{\frac{n}{\log n}}\right)$ slots.

We will also argue that in another $O\left(\sqrt{\frac{n}{\log n}}\right)$ number of slots, the value of $\mathcal{Z}$ would have diffused to each node of the network. Note that the best one-shot protocol in an organized network, under this choice of $r_{n}$ will also require $\Theta\left(\sqrt{\frac{n}{\log n}}\right)$ time slots for a one-shot computation of MAX. The bound on the time in Theorem 1 is therefore tight.

\subsection{Pipelined computation of MAX using Aloha}

If $\mathcal{Z}$ were to be computed continuously using the One-Shot MAX protocol, a throughput of $\Theta\left(\sqrt{\frac{\log n}{n}}\right)$ can be achieved. We believe some structure in the network is necessary to do better. We will assume that all nodes have a transmission range that is exactly $r_{n}$. This strict requirement can be easily relaxed but we will keep this assumption for pedagogical convenience.

We impose the following structure in the network. Prior to the computation, each node obtains its minimum hop distance to the sink. Henceforth, we will refer to this as simply the hop distance of 
the node. From (1), each cell in the tessellation is occupied. Since nodes in adjacent cells differ in their hop distance by atmost 1 , the largest hop distance of a node in the network is no more than $d:=2 l_{n}=2\left\lceil\sqrt{\frac{n}{2.75 \log n}}\right\rceil$.

Let $h_{i}$ be the hop distance of Node $i$. Observe that a transmission by Node $i$ can be decoded successfully by Node $j$ only if $\left|h_{i}-h_{j}\right| \leq 1$. Hence, if there is a reception by Node $i$ in slot $t$, then that transmission must have been made by a node with hop distance either $\left(h_{i}-1\right), h_{i}$, or $\left(h_{i}+1\right)$. Thus, if a node transmits its hop distance modulo 3 along with its transmitted bit, then every receiver that can decode this transmission successfully, can also, by the receiver's knowledge of its own hop distance, correctly identify the hop distance of the transmitter.

Time is divided into rounds, where each round consists of $\tau$ slots. Minimizing $\tau$ would maximize the throughput. We will discuss this in Section 3 . Data arrives at each node at the beginning of each round, that is, at the rate of 1 data bit per round. Let the value of the bit at Node $i$ in the round $r$ be $Z_{i}(r)$. $\mathcal{Z}(r):=\max _{1 \leq i \leq n} Z_{i}(r)$, for $r=1,2, \ldots$, is to be made available at the sink node, Node $s$.

Pipelined MAX protocol is as follows. The sink only receives data and does not transmit. The other nodes in the network perform the following. (We remind here that the naming of the nodes is for our convenience. The nodes themselves do not know their identity.)

In each slot, Node $i$ either transmits with probability $p$ or listens with probability $(1-p)$ independently of all other transmissions in the network. The value of $p$ is chosen as in the One-Shot MAX protocol. Each node executes the following protocol for round $r$.

Transmission: If Node $i$ transmits in slot $t$ of round $r$, then it transmits three bits $\left(A_{i}, B_{i}, T_{i}(r, t)\right)$ in the slot. Bits $A_{i}$ and $B_{i}$ are the identification bits and are obtained as $\left(h_{i} \bmod 3\right)$. The bit $T_{i}(r, t)$ is the transmitted data bit and is obtained as

$$
T_{i}(r, t)=\max \left\{Z_{i}\left(r-d+h_{i}\right), Y_{i}(r-1)\right\}
$$

Here, by convention, $Z_{i}(v)=Y_{i}(v)=0$ for $v \leq 0 . Y_{i}(r-1)$ is computed from succesful receptions in round $(r-1)$, as described below.

Reception: In round $r$, Node $i$ maintains $Y_{i}(r, t)$ for $t=0,1,2, \ldots, \tau . Y_{i}(r, 0)$ is initialized to 0 at the beginning of round $r$. $Y_{i}(r, t)$ stores the MAX of the data bits that Node $i$ has decoded from all the slots in round $r$, upto and including slot $t$, and which were transmitted by the nodes with hop distance $\left(h_{i}+1\right)$. In slot $t$ of round $r$, if Node $i$ successfully receives a transmission from a node with hop distance $\left(h_{i}+1\right)$ (available from the identification bits), then it sets the received data bit to be $X_{i}(r, t)$. If Node $i$ senses an idle or a collision in slot $t$, or if it receives a successful 
transmission from a node with hop distance different from $\left(h_{i}+1\right)$, then it sets $X_{i}(r, t)=0$. Thus, $Y_{i}(r, t)=\max \left\{Y_{i}(r, t-1), X_{i}(r, t)\right\}$. Define $Y_{i}(r):=Y_{i}(r, \tau)$.

The sink node, Node $s$, obtains the MAX as $\mathcal{Z}(r-d)=\max \left\{Z_{s}(r-d), Y_{s}(r)\right\}$, for all $r>d$. The delay of the protocol is $d$ rounds or $d \tau$ time slots.

Theorem 2 If all the nodes execute the protocol Pipelined MAX, then for any $\alpha, k>0$, there exists $\tau=\tau(\alpha, k)=\Theta\left(\log ^{2} n\right)$ so that the correct MAX is available at the sink in a round with probability atleast $\left(1-\frac{k}{n^{\alpha}}\right)$. This achieves a throughput of $\Omega\left(\frac{1}{\log ^{2} n}\right)$ with a delay of $O\left(\sqrt{n \log ^{3} n}\right)$ slots.

The optimal pipelined protocol for MAX in an organized network requires $\Theta(\log n)$ slots for each round in the absence of block coding. Thus, the penalty for minimal organization and no coordination is the $\log n$ overhead for the length of each round. Also, for our protocol, Node $i$, with a hop distance of $h_{i}$, requires a memory of $\left(d-h_{i}+1\right)$ bits to store $Z_{i}(r), Z_{i}(r-1), \ldots, Z_{i}\left(r-d+h_{i}\right)$. Thus, the protocol requires each node to have $(d+1)$ bits of memory for storage of past data values.

\section{Proofs}

\subsection{Preliminaries}

\subsubsection{Bounding the Number of Interfering Neighbors}

Define the interfering neighborhood of Node $i$ by $\mathcal{N}_{i}^{(I)}:=\left\{j: 0<\left\|X_{i}-X_{j}\right\| \leq(1+\Delta) r_{n}\right\}$. As discussed earlier, a transmission from Node $i$ in slot $t$ is deemed successful if all nodes within $r_{n}$ of Node $i$ can decode this transmission without a collision. A sufficient condition for Node $i$ to be successful in transmitting in slot $t$ is that no node belonging to $\mathcal{N}_{i}^{(I)}$ must transmit in slot $t$.

From the protocol model, the choice of $s_{n}$ and (1), the set of nodes that interfere with a transmission from a node in Cell $c$, (i.e., $\bigcup_{i \in S_{c}} \mathcal{N}_{i}^{(I)}$ ) is contained within an interference square centered at Cell $c$. This square contains $k_{1}=\left(2\left\lceil\frac{(1+\Delta) r_{n}}{s_{n}}\right\rceil+1\right)^{2}$ cells. From (1),

$$
\left|\mathcal{N}_{i}^{(I)}\right| \leq k_{1} c_{2} \log n-1
$$

Observe that $k_{1}$ is a constant for large enough $n$. 


\subsubsection{Probability of a successful transmission from a cell}

Let $P_{i}$ be the probability that Node $i$ transmits successfully in a slot and $P^{(c)}$, the probability that some node in Cell $c$ transmits successfully in a slot. $P_{i} \geq p(1-p)^{\left|\mathcal{N}_{i}^{(I)}\right|}$, and from (2), we have $P_{i} \geq p(1-p)^{k_{1} c_{2} \log n-1}$. Successful transmissions by nodes from Cell $c$ are mutually disjoint events, and hence, $P^{(c)}=\sum_{i \in S_{c}} P_{i} \geq N_{c} p(1-p)^{k_{1} c_{2} \log n-1}$. From (1), we have $N_{c} \geq c_{1} \log n \forall c \in \mathcal{C}$ and hence, $P^{(c)} \geq c_{1} \log n p(1-p)^{k_{1} c_{2} \log n-1}$. Choosing $p=\frac{1}{k_{1} c_{2} \log n}$ maximises the lower bound in this inequality and yields

$$
P^{(c)} \geq \frac{c_{1}}{k_{1} c_{2}}\left(1+\frac{1}{k_{1} c_{2} \log n-1}\right)^{-\left(k_{1} c_{2} \log n-1\right)} \geq \frac{c_{1}}{k_{1} c_{2} e}=: p_{S}
$$

Thus, the probability of successful transmission from a cell is lower bounded by a constant $p_{S}$, independent of the number of nodes in the network. This will be crucial to our analysis.

\subsection{Proof of Theorem 1}

We will prove Theorem 1 by proving bounds on the total time required by each of phases I, II and III.

\subsubsection{Phase I: Data aggregation within each cell}

Consider Cell $c$. Let $\mathcal{T}_{c}$ be the total number of slots required for every node in Cell $c$ to have transmitted successfully atleast once. Recall that $p=\left(k_{1} c_{2} \log n\right)^{-1}$. We will bound $\mathcal{T}_{c}$ by stochastic domination. Consider a sample space $\mathcal{S}$ containing mutually disjoint events $E_{1}, E_{2}, \ldots, E_{N_{c}}$. Let $\operatorname{Pr}\left(E_{q}\right)=p(1-p)^{k_{1} c_{2} \log n-1}$ for $1 \leq q \leq N_{c}$. Observe that $P_{i} \geq \operatorname{Pr}\left(E_{q}\right) \forall i \in S_{c}$ and $1 \leq q \leq N_{c}$. Let $E=\bigcup_{q=1}^{N_{c}} E_{q}$. We have $P_{E}:=\operatorname{Pr}(E)=N_{c} p(1-p)^{k_{1} c_{2} \log n-1}$. Let a sequence of samples be drawn independently from $\mathcal{S}$. Let the number of samples required to be drawn from $\mathcal{S}$ so that each of the events $E_{q}, q=1,2, \ldots N_{c}$ occurs atleast once, be the random variable $T_{c}^{\prime}$. The probability of occurence of $E$ in a given sample is $P_{E}$ and hence, the waiting time in terms of number of samples drawn, for the event $E$ to occur, as well as the waiting time between consecutive occurences of $E$, is given by the geometrically distributed random variable $\operatorname{Geom}\left(P_{E}\right)$. Now, consider the events of successful occurences of event $E$. If $(l-1)$ distinct events among $E_{q}, 1 \leq q \leq N_{c}$ have already occured, then the probability that the next occurence of $E$ is due to an as yet unoccured event $E_{q^{\prime}}$ is $\left(1-\frac{l-1}{N_{c}}\right)$, as each $E_{q}, 1 \leq q \leq N_{c}$ is equally probable. The number of occurences of event $E$ to wait for the occurence of an as yet unoccured event among $E_{q}, 1 \leq q \leq N_{c}$ is distributed 
as $\operatorname{Geom}\left(1-\frac{l-1}{N_{c}}\right)$. The random variable $T_{c}^{\prime}$ can thus, be expressed as: $T_{c}^{\prime}=\sum_{j=1}^{R_{c}^{\prime}} t_{c, j}^{\prime}$ where $t_{c, j}^{\prime} \sim \operatorname{Geom}\left(P_{E}\right)$ and $R_{c}^{\prime} \sim \sum_{l=1}^{N_{c}} \operatorname{Geom}\left(1-\frac{l-1}{N_{c}}\right)$.

Now compare the following two events: (1) Event $\mathcal{A}$ defined as the successful transmission from Cell $c$ resulting from a successful transmission by Node $i$ in Cell $c$ and (2) Event $\mathcal{B}$ defined as the occurrence of $E$ in a sample drawn from $\mathcal{S}$ due to the occurence of $E_{q}$. Observe that $\operatorname{Pr}(\mathcal{A}) \geq \operatorname{Pr}(\mathcal{B})$. From this comparison, we see that $\mathcal{T}_{c}$ will be stochastically dominated by $T_{c}^{\prime}$ i.e. $\operatorname{Pr}\left(\mathcal{T}_{c} \geq z\right) \leq$ $\operatorname{Pr}\left(T_{c}^{\prime} \geq z\right) \forall z \in \mathbb{N}$. Further, $T_{c}^{\prime}$ will be stochastically dominated by the random variable $T_{c}=$ $\sum_{j=1}^{R_{c}} t_{c, j}$, where $t_{c, j} \sim \operatorname{Geom}\left(p_{S}\right)$ and $R_{c} \sim \sum_{l=1}^{m} \operatorname{Geom}\left(1-\frac{l-1}{m}\right)$ with $m=\left\lceil c_{2} \log n\right\rceil$ which is an upper bound on $N_{c}$ from (1). We therefore, have

$$
\operatorname{Pr}\left(\mathcal{T}_{c} \geq z\right) \leq \operatorname{Pr}\left(T_{c} \geq z\right) \forall z \in \mathbb{N}
$$

It is convenient to work with the random variable $T_{c}$ because it is independent of the parameters of Cell $c$. We will obtain the moment generating functions (mgf) of the distributions of the integervalued random variables involved. Let the mgf of each random variable be denoted by the same character in sans serif font. For a random variable $F, \operatorname{F}(z)=\sum_{j \in \mathbb{Z}} \operatorname{Pr}(F=j) z^{-j}$. The region of convergence of the mgf is specified in parentheses.

$$
\begin{aligned}
\mathrm{t}_{\mathrm{c}, \mathrm{j}}(z) & =\frac{p_{S} z^{-1}}{1-\left(1-p_{S}\right) z^{-1}}:=S(z) \quad\left(|z|>1-p_{S}\right) \\
\mathrm{R}_{\mathrm{c}}(z) & =\prod_{l=1}^{m} \frac{\left(1-\frac{l-1}{m}\right) z^{-1}}{1-\frac{l-1}{m} z^{-1}} \quad\left(|z|>1-\frac{1}{m}\right) \\
\mathrm{T}_{\mathrm{c}}(z) & =\sum_{r \in \mathbb{N}} \operatorname{Pr}\left(R_{c}=r\right)[S(z)]^{r} \\
& =\mathrm{R}_{\mathrm{c}}\left(\frac{1}{S(z)}\right) \quad m ! p_{S}^{m} \\
& =\frac{\Pi_{l=1}^{m}\left(m\left[z-\left(1-p_{S}\right)\right]-(l-1) p_{S}\right)}{\left(|z|>1-\frac{p_{S}}{m}\right)}
\end{aligned}
$$

Thus, $\mathbb{E}\left[e^{s T_{c}}\right]=\frac{m ! p_{S}^{m}}{\Pi_{l=1}^{m}\left(m\left[e^{-s}-\left(1-p_{S}\right)\right]-(l-1) p_{S}\right)}$ for $s<\log \left(\frac{1}{1-\frac{p_{S}}{m}}\right)$. Choose $s_{1}=\log \left(\frac{1}{1-\frac{p_{S}}{2 m}}\right)$. After some algebra, we can show the following.

$$
\begin{aligned}
\mathbb{E}\left[e^{s_{1} T_{c}}\right] & =\frac{m ! p_{S}^{m}}{m^{m}} \Pi_{l=1}^{m}\left(e^{-s_{1}}-1+\frac{m-l+1}{m} p_{S}\right)^{-1} \\
& =c_{m} \sqrt{\pi m}
\end{aligned}
$$


Here $c_{m}=\frac{2^{2 m}}{\left(\begin{array}{c}2 m \\ m\end{array}\right) \sqrt{\pi m}} \rightarrow 1$ as $m \rightarrow \infty$ by the Stirling approximation. From the Chernoff bound we get $\operatorname{Pr}\left(\mathcal{T}_{c} \geq V_{1}\right) \leq \operatorname{Pr}\left(T_{c} \geq V_{1}\right) \leq c_{m} \sqrt{\pi m}\left(1-\frac{p_{S}}{2 m}\right)^{V_{1}}$. By the union bound, we have

$$
\operatorname{Pr}\left(\max _{c \in \mathcal{C}} \mathcal{T}_{c} \geq V_{1}\right) \leq M_{n} c_{m} \sqrt{\pi m}\left(1-\frac{p_{S}}{2 m}\right)^{V_{1}}
$$

To achieve $\operatorname{Pr}\left(\max _{c \in \mathcal{C}} \mathcal{T}_{c} \geq V_{1}\right) \leq \frac{k}{n^{\alpha}}$, it is sufficient to have $\left(1-\frac{p_{S}}{2 m}\right)^{V_{1}} \leq \frac{k}{n^{\alpha} M_{n} c_{m} \sqrt{\pi m}}$ or

$$
V_{1} \geq \frac{\frac{1}{2} \log m+\log M_{n}+\alpha \log n-\log k+\frac{1}{2} \log \pi+\log c_{m}}{-\log \left(1-\frac{p_{S}}{2 m}\right)}
$$

Here, $m=\left\lceil c_{2} \log n\right\rceil, M_{n}=\left\lceil\sqrt{\frac{n}{2.75 \log n}}\right\rceil^{2}$. Writing $-\log \left(1-\frac{p_{S}}{2 m}\right)=\frac{p_{S}}{2 m}+\frac{p_{S}^{2}}{2(2 m)^{2}}+\ldots$, we can see that there exists a choice of $V_{1}=O\left(\log ^{2} n\right)$, which would be sufficient for the completion of Phase I, i.e., every node in every cell of the network would have successfully transmitted at least once in $V_{1}$ slots, with probability at least $\left(1-\frac{k}{n^{\alpha}}\right)$.

\subsubsection{Phase II: Progress to the bottom of the square}

Let the columns of cells shown in Fig. 1 be numbered $C_{1}, C_{2}, \ldots, C_{l_{n}}$. Let the $l_{n}$ cells in each column be numbered from 1 to $l_{n}$ from top to bottom. In this phase, we are concerned with transmissions in the top $w:=l_{n}-1$ cells of each column. In Phase I, each node has successfully received the transmissions by every other node in its cell. Hence, Phase II will be completed if the following sequence of events occurs for each column $C$ : A successful transmission by some node in the first cell of the column, followed by a successful transmission by some node in the second cell of the column and so on until a successful transmission by some node in the $w$-th cell of the column.

Let the number of slots required for this sequence of events be $\mathcal{T}^{(C)}$ for column $C$. We can see that $\mathcal{T}^{(C)}$ will be stochastically dominated by $T^{(C)}:=\sum_{j=1}^{w} t_{j}^{(C)}$, where $t_{j}^{(C)} \sim \operatorname{Geom}\left(p_{S}\right)$. We can thus 
derive the following.

$$
\begin{aligned}
\mathrm{T}^{(\mathrm{C})}(z) & =\frac{p_{S}^{w} z^{-w}}{\left(1-\left(1-p_{S}\right) z^{-1}\right)^{w}} \\
\mathbb{E}\left[e^{\left.s T^{(C)}\right]}\right. & =\frac{\left(|z|>1-p_{S}\right)}{\left(e^{-s}-\left(1-p_{S}\right)\right)^{w}} \\
& \text { for } s<\log \left(\frac{1}{1-p_{S}}\right) \\
\operatorname{Pr}\left(T^{(C)} \geq V_{2}\right) \leq & \frac{\mathbb{E}\left[e^{s_{2} T^{(C)}}\right]}{e^{s_{2} V_{2}}}=2^{w}\left(1-\frac{p_{S}}{2}\right)^{V_{2}} \\
\operatorname{Pr}\left(\max _{1 \leq j \leq l_{n}} \mathcal{T}^{\left(C_{j}\right)} \geq V_{2}\right) \leq & l_{n} 2^{w}\left(1-\frac{p_{S}}{2}\right)^{V_{2}}
\end{aligned}
$$

where we have used $s_{2}=\log \left(\frac{1}{1-\frac{p_{S}}{2}}\right)$ in the Chernoff bound. Thus, to achieve $\operatorname{Pr}\left(\max _{1 \leq j \leq l_{n}} T^{\left(C_{j}\right)} \geq V_{2}\right) \leq$ $\frac{k}{n^{\alpha}}$, it suffices to have $\left(1-\frac{p_{S}}{2}\right)^{V_{2}^{2}} \leq \frac{k}{n^{\alpha} l_{n} 2^{w}}$ or

$$
V_{2} \geq \frac{\alpha \log n+\log l_{n}+w \log 2-\log k}{-\log \left(1-\frac{p_{S}}{2}\right)}
$$

Now, $l_{n}=\left\lceil\sqrt{\frac{n}{2.75 \log n}}\right\rceil=w+1$, and hence, $V_{2}=O\left(\sqrt{\frac{n}{\log n}}\right)$ slots are sufficient for the completion of Phase II with probability at least $\left(1-\frac{k}{n^{\alpha}}\right)$.

\subsubsection{Phase III: Progress into the sink}

Phase III comprises diffusion of the MAX into the cell containing the sink. Let the time required for this to happen be the random variable $T_{s}$. It is easily seen from the analysis of the sequence of transmission for Phase II that $\operatorname{Pr}\left(T_{s} \geq V_{3}\right) \leq 2^{w}\left(1-\frac{p_{S}}{2}\right)^{V_{3}}$ where $w$ is as defined before. Calculations similar to those in the analysis for Phase II show that $V_{3}=O\left(\sqrt{\frac{n}{\log n}}\right)$ slots are sufficient for completion of this phase with probability at least $\left(1-\frac{k}{n^{\alpha}}\right)$.

\subsubsection{Bound on the overall time}

Since each of phases I, II and III get completed in $O\left(\sqrt{\frac{n}{\log n}}\right)$ time slots with probability at least $\left(1-\frac{k^{\prime}}{n^{\alpha}}\right)$, for appropriate constants $k^{\prime}$, the protocol One-Shot MAX achieves computation of the MAX at the sink in $O\left(\sqrt{\frac{n}{\log n}}\right)$ number of time slots with probability at least $\left(1-\frac{k}{n^{\alpha}}\right)$. If the protocol is followed for another $V_{3}+V_{2}$ slots, the true MAX will diffuse to the complete bottom row, 
and then to the complete network, the direction of diffusion being opposite to that in Phase III and Phase II respectively.

\subsection{Obtaining the Hop Distance}

The following algorithm Hop Distance Compute obtains the hop distance for each node in the network. $\lceil\log d\rceil$ slots are grouped into a frame and $\tau=\Theta\left(\log ^{2} n\right)\left(\tau=V_{1}\right.$ as obtained in Phase I analysis of protocol One-Shot MAX) frames form a superframe. The algorithm ends after $(d+1)$ superframes.

Let the superframes be denoted by $g_{0}, g_{1}, \ldots, g_{d}$. A node either transmits in every slot of a frame or it does not transmit in any slot of the frame. Each transmission is a number expressed in $\lceil\log d\rceil$ bits. At the beginning of the algorithm, the sink transmits the number 0 expressed in $\lceil\log d\rceil$ bits in each frame of superframe $g_{0}$. Each node of the network other than the sink executes the following algorithm. Node $i$ makes no transmission till it has decoded a transmission successfully. Let the first successful reception by Node $i$ happen in a frame belonging to superframe $g_{i}$ and let the decoded transmission correspond to the number $n_{i}$ expressed in $\lceil\log d\rceil$ bits. Node $i$ sets its hop distance to $\left(n_{i}+1\right)$ and ignores other successfully received bits in frames from superframe $g_{i}$. During the $\tau$ frames from superframe $g_{i+1}$, Node $i$ transmits, in each frame, the number $\left(n_{i}+1\right)$ expressed in $\lceil\log d\rceil$ bits, with probability $p$, independently of all the other transmissions in the network and makes no transmission with probability $(1-p)$. After the end of round $g_{i+1}$, Node $i$ makes no more transmissions. The total number of slots required is $(d+1) \tau\lceil\log d\rceil$.

Lemma 1 The nodes of the network correctly compute their minimum hop distance from the sink, using Hop Distance Compute in $O\left(\sqrt{n \log ^{5} n}\right)$ time slots with probability at least $\left(1-\frac{k}{n^{\alpha}}\right)$ for any positive $\alpha$ and some constant $k$.

We omit the proof of this lemma.

\subsection{Proof of Theorem 2}

Let the set of nodes at hop distance $h$ be $G_{h}$. Let $t_{i, r}$ be the first slot in round $r$ that Node $i$ transmits succesfully in. The number of slots in a round is $\tau=\Theta\left(\log ^{2} n\right)\left(\tau=V_{1}\right.$ from Phase I). Every node in the network would have transmitted successfully at least once in each round of $\tau$ slots w.h.p. Let $h_{\max } \leq d$ be the largest hop distance of a node in the network. In the proof, we will assume that each node of the network transmits successfully in each round at least once. We 
claim that

$$
\max _{i \in G_{h}} T_{i}\left(r, t_{i, r}\right)=\max _{j \in \bigcup_{h \leq f \leq d} G_{f}} Z_{j}(r-d+h)
$$

for $0 \leq h \leq h_{\max }$ and $r>d-h$. The sink being at hop distance 0 , proving the claim will complete the proof. Assume that the claim is true for $h_{0}<h \leq h_{\max }$ for $r>d-h$. We shall show that the claim will then be true for $h=h_{0}$ and for $r>d-h_{0}$. Consider transmissions by the nodes at hop distance $h_{0}$ in round $(r+1)$.

$$
\max _{i \in G_{h_{0}}} T_{i}\left(r+1, t_{i, r+1}\right)=\max _{i \in G_{h_{0}}}\left\{\max \left\{Z_{i}\left(r+1-d+h_{0}\right), Y_{i}(r)\right\}\right\}
$$

Since each node at hop distance $\left(h_{0}+1\right)$ transmits successfully at least once in round $r$, the transmission of each such node is decoded successfully by some node at hop distance $h_{0}$. Hence,

$$
\begin{aligned}
\max _{i \in G_{h_{0}}} Y_{i}(r) & =\max _{j \in G_{h_{0}+1}} T_{j}\left(r, t_{j, r}\right) \\
& =\max _{j \in \bigcup_{h_{0}+1 \leq f \leq d} G_{f}} Z_{j}\left(r-d+h_{0}+1\right)
\end{aligned}
$$

where the second equality follows from the induction hypothesis. Hence,

$$
\begin{array}{r}
\max _{i \in G_{h_{0}}} T_{i}\left(r+1, t_{i, r+1}\right)=\max \left\{\max _{i \in G_{h_{0}}} Z_{i}\left(r+1-d+h_{0}\right),\right. \\
\left.\max _{j \in \cup_{h_{0}+1 \leq f \leq d} G_{f}} Z_{j}\left(r-d+h_{0}+1\right)\right\} \\
\left.=\max _{j \in \cup_{h_{0} \leq f \leq d} G_{f}} Z_{j}\left(r-d+h_{0}+1\right)\right\}
\end{array}
$$

which proves the claim for hop distance $h_{0}$ for round $(r+1)$. By induction, the claim is true for each $h$ and each round $r>d-h$. Therefore, the sink Node $s$ correctly sets $\mathcal{Z}(r-d)=\max \left\{Z_{s}(r-d), Y_{s}(r)\right\}$. The delay of the protocol is $d \tau=\Theta\left(\sqrt{n \log ^{3} n}\right)$ slots.

As transmissions by different nodes are independent, the analysis in the diffusion of phase I of One-Shot MAX carries over. The probability that the computed value of $\mathcal{Z}(r)$ is incorrect for any given round is upper bounded by $\frac{k}{n^{\alpha}}$ for any constants $\alpha, k>0$. 


\section{Discussion}

The total number of transmissions (successful as well as unsuccessful) in one execution of One-Shot

MAX is $\Theta\left(\frac{n^{3 / 2}}{\log ^{3 / 2} n}\right)$. In Pipelined MAX, a total of $\Theta(n \log n)$ transmissions are made per round. Note that the corresponding number is $\Theta(n)$ with a coordinated protocol for both cases.

Our analysis can be extended to the case where the nodes use pure Aloha as the MAC. We need to use a transmission rate rather than a transmission probability. The success probabilities are calculated similarly except that we now have a collision window that is twice the packet length. All calculations are analogous.

It is fairly straightforward to show that in a noiseless, structure-free broadcast network, the histogram can be computed in $\Theta(n)$ slots w.h.p. In the noisy broadcast network, by a simple modification of the protocol of [1], we can show that the histogram can be computed in $\Theta(n \log \log n)$ slots w.h.p.

\section{References}

[1] R. G. Gallager. Finding parity in simple broadcast networks. IEEE Trans. on Info. Theory, 34:176-180, 1988.

[2] A. Giridhar and P. R. Kumar. Computing and communicating functions over sensor networks. IEEE Journal on Selected Areas in Communications, 23(4):755-764, April 2005.

[3] P. Gupta and P. R. Kumar. Critical power for asymptotic connectivity in wireless networks. In W. M. McEneaney, G. Yin, and Q. Zhang, editors, Stochastic Analysis, Control, Optimization and Applications: A Volume in Honor of W. H. Fleming. Birkhauser, Boston, 1998.

[4] Y. Kanoria and D. Manjunath. On distributed computation in noisy random planar networks. In Proc. of IEEE ISIT, Nice, France, June 2007.

[5] N. Khude, A. Kumar, and A. Karnik. Time and energy complexity of distributed computation in wireless sensor networks. In Proceedings of IEEE INFOCOM, pages 2625-2637, 2005.

[6] E. Kushilevitz and Y. Mansour. Computation in noisy radio networks. In Proc. of SODA, pages 236-243, 1998.

[7] F. Xue and P. Kumar. The number of neighbors needed for connectivity of wireless networks. Wireless Networks, 10(2):169-181, March 2004. 